\title{
PROPOSTA DE BSC PARA INSTITUIÇÃO DE ENSINO SUPERIOR PRIVADA SOB A PERSPECTIVA DE CLIENTES
}

\author{
Ana Paula Archer de Arruda Borges ${ }^{1}$ Evandro Vieira Cabral $^{2}$ Sergio Murilo Petri ${ }^{3}$ \\ Universidade Federal de Santa Catarina - UFSC ${ }^{1}$, Faculdades Borges de Mendonça ${ }^{2}$, Universidade Federal de Santa \\ Catarina - UFSC ${ }^{3}$ \\ anaborges00@hotmail.com, evandro.cabral@bm.edu.br, smpetri@gmail.com
}

\section{Resumo}

O objetivo deste artigo é propor indicadores de avaliação de desempenho da Perspectiva de Clientes por meio da metodologia do Balanced Scorecard (BSC) para uma Instituição de Ensino Superior Privada (IES) do Brasil. A elevada concorrência no ramo da educação superior privada justifica a necessidade destas instituições possuírem estratégias bem definidas e agilidade para acompanhar resultados e tomar decisões. Este artigo, com abordagem qualitativa, trata-se de uma pesquisa bibliográfica e documental e um estudo de caso em uma IES privada da cidade de Florianópolis, SC. Por meio do BSC foram apontados indicadores como "imagem da instituição perante a sociedade", "índice de empregabilidade dos estudantes" e "satisfação dos estudantes" que foram citados também nos trabalhos dos autores pesquisados no levantamento bibliométrico, evidenciando semelhanças nos resultados alcançados. $O$ "nível de preços dos serviços educacionais" foi o único indicador não citado nas pesquisas estudadas. A definição de indicadores de performance para a Perspectiva dos Clientes evidenciaram a necessidade de aproveitar oportunidades estratégicas até então ignoradas pela instituição estudada.

Palavras-chave: IES privada. Balanced Scorecard. Perspectiva de clientes. Avaliação de desempenho.

\section{PROPOSAL OF BSC FOR PRIVATE INSTITUTION OF HIGHER EDUCATION UNDER THE CUSTOMER'S PERSPECTIVE}

\begin{abstract}
The objective of this paper is to propose indicators for evaluating the performance of Customer Perspective through the methodology of Balanced Scorecard (BSC) for a Private Institution of Higher Education from Brazil. The high competition in the field of private higher education justifies the need for these institutions to have well-defined strategies and agility to track results and make decisions. This article with a qualitative approach, is a bibliographic and documentary research and a case study at a private IHE in the city of Florianópolis, SC. Using the BSC, indicators were identified as "image of the institution in society", "employability index of students" and "student satisfaction" that were also mentioned in the works of authors researched in bibliometric survey, showing similarities in the results achieved. The "price level of educational services" was the only indicator not mentioned in the research studied. The definition of performance indicators for the Customer Perspective highlighted the need to take advantage of strategic opportunities until then ignored by the institution studied.
\end{abstract}

Keywords: Private institution of higher education. Customer perspective. Balanced Scorecard. Performance evaluation. 


\section{Introdução}

O Ensino Superior, no Brasil, desde a década de 30 vem evoluindo e crescendo significativamente. Estatísticas gerais da educação superior brasileira apresentam dados que demonstram mudanças importantes e essenciais para o desenvolvimento do país.

A evolução da Educação Superior Brasileira é traduzida nos dados do Censo da Educação Superior de 2011, divulgado pelo Ministério da Educação em parceria com o INEP (Instituto Nacional de Estudos e Pesquisas Educacionais Anísio Teixeira). Em 2011 foram quase sete milhões de matrículas na educação superior de graduação, comparados com aproximadamente 1,5 milhões em 1980. Além do crescimento em quantidade de matrículas, a proporção entre Instituições de Ensino Superior (IES) privadas e públicas também sofreu significativa mudança. Tal relação, segundo o Censo, demonstra que em 2011 as IES privadas tiveram uma participação de $73,7 \%$ no total de matrículas de graduação.

Segundo Barreyro (2008), o sistema Federal de ensino superior está integrado pelas IES mantidas pela União e as criadas e mantidas pela iniciativa privada. São consideradas públicas as instituições criadas ou incorporadas, mantidas e administradas pelo poder público. São consideradas privadas, as mantidas e administradas por pessoas físicas ou jurídicas de direito privado.

Este estudo se justifica pelo alto crescimento das iniciativas privadas no ramo da educação, que passaram a se tornar atores importantes na universalização do ensino superior oferecido no país. Em um cenário onde há grande predomínio de instituições privadas, são necessárias estratégias bem definidas e agilidade no acompanhamento dos resultados e na avaliação de desempenho destas organizações. Manter-se num mercado altamente competitivo que enfrenta avaliações constantes, obriga gestores e empreendedores a tomar decisões rápidas e corretas.

Para Ramos e Lobo (2010), a sobrevivência das IES, principalmente de pequeno porte, depende da capacidade de se adaptar e agir em resposta às pressões ambientais. A estratégia é um conjunto de medidas e decisões estabelecidas para serem tomadas e executadas no tempo e hora certa, com o propósito de conduzir a empresa, neste caso a mantenedora, aos objetivos propostos pelos seus dirigentes da mantida e da mantenedora.

Diversas são as ferramentas de gestão para monitorar o cumprimento das estratégias e que auxiliam na avaliação e análise de resultados e desempenho. O Balanced Scorecard (BSC) é uma metodologia de medição e gestão de desempenho criada por dois professores da Harvard Business School, Robert Kaplan e David Norton, desenvolvido como um modelo de avaliação e performance empresarial voltado para a gestão estratégica organizacional sob a ótica de aprendizado e crescimento, processos internos, clientes e financeira. Este estudo se aprofundará na perspectiva dos clientes (stakeholders), traduzida como as relações da organização com o mercado, clientes e potenciais clientes.

Diante do exposto surge o seguinte problema de pesquisa: Quais indicadores seriam adequados para a Perspectiva dos Clientes, da metodologia do $B S C$, em uma IES privada?

O objetivo principal deste estudo é propor indicadores de avaliação de desempenho da Perspectiva de Clientes da metodologia do $B S C$ para uma IES privada. Para tanto serão traçados alguns objetivos específicos como (i) Pesquisar a experiência internacional de adoção do $B S C$ no ensino superior nos últimos dez anos, (ii) aprofundar a perspectiva de clientes na instituição estudada e (iii) propor indicadores relevantes para uma IES privada .

O tema da pesquisa foi escolhido em função do crescimento significativo das IES privadas no ensino superior brasileiro que necessitam de uma ferramenta de gestão eficiente capaz de atender suas especificidades.

Este artigo está dividido em cinco seções sendo a próxima a revisão bibliográfica em que é apresentada a teoria necessária para a compreensão deste estudo, a terceira seção é apresentada a 
metodologia da pesquisa, a quarta são evidenciados os resultados e por fim as considerações finais desta pesquisa.

\section{Revisão bibliográfica}

Este capítulo abordará o referencial teórico necessário para o desenvolvimento deste artigo.

\subsection{Avaliação de desempenho}

A competitividade e a rapidez com que as informações circulam nos dias atuais exigem que as organizações tenham controle e segurança para a tomada de decisão. A segurança citada pode estar na certeza de que as metas estão sendo alcançadas e que o planejamento da empresa está sendo concretizado a cada dia, ou seja, o desempenho da organização está de acordo com objetivos por ela traçado.

Para Galvão, Corrêa e Alves (2011), tomar decisões em um ambiente competitivo e de forte pressão exige dos executivos a capacidade para o planejamento e controle do negócio. Os níveis organizacionais devem responder, com agilidade e flexibilidade, ao processo de comunicação e tomada de decisão. Por outro lado, a tomada de decisão pode não ser tarefa fácil, pois a empresa é um organismo dinâmico que interage constantemente com o ambiente.

Os mesmos autores afirmam ainda que aspectos como confiança e compartilhamento de ideias reforçam a adoção da avaliação do desempenho organizacional para o gerenciamento dos negócios da empresa. A utilização de técnicas para medir e monitorar o desempenho organizacional é crítica, e o processo de tomada de decisão requer qualidade e agilidade das informações.

Para Rigby, Bridelli e Alves (2003), o aumento na demanda por ferramentas de gestão que auxiliam na avaliação de desempenho das organizações ocorre por diversos fatores, entre eles o aumento das expectativas por parte dos acionistas, pressão contínua para redução de custos, necessidade de crescimento, necessidade de encarar períodos de recessão e a obrigação dos executivos em apresentar resultados de forma consistente. Os autores levantaram as ferramentas mais utilizadas no mundo em 2003, são elas: Planejamento Estratégico, Benchmarking, Missão e Visão, Segmentação da Base de Clientes, Outsourcing, Pesquisas Junto ao Cliente, Gerenciamento da Relação com o Cliente (CRM), Códigos de Ética Corporativa, Estratégias de Crescimento, Remuneração por Desempenho, Competências Essenciais, Planejamento para Contigências, Alianças Estratégicas, Programa de Gerenciamento de Mudanças, Knowledge Management, Integração Logística, Downsizing, Gerenciamento da Qualidade Total, Reengenharia, Painel de Controle (Balanced Scorecard), Análise de Valor Econômico Agregado, Gerenciamento Baseado na Atividade, Equipes de Integração Pós Fusão, Corporate Venturing, Stock Buybacks. Os autores ainda afirmam que toda a ferramenta carrega um conjunto de pontos forte e fracos. O êxito na sua aplicação requer o entendimento de todos os efeitos (inclusive colaterais) de cada uma.

A pesquisa em questão utilizará a ferramenta conhecida como Balanced Scorecard. Tal escolha deu-se pelo fato deste método estar bastante em evidência, abordar as áreas de maior interesse dentro das organizações e atender às necessidades de curto e longo prazo das instituições. A seguir serão tratados os aspectos do Balanced Scorecard.

\subsection{A abordagem do Balanced Scorecard (BSC)}

A abordagem do Balanced Scorecard foi proposta por Robert Kaplan e David Norton, em artigo publicado na Harvard Business Review, na edição de janeiro-fevereiro de1992.

Segundo os autores, os modelos de gestão tradicionalmente utilizados pelas corporações estão atrelados a indicadores da contabilidade financeira, que analisam apenas os ativos tangíveis e 
refletem basicamente os resultados de curto prazo obtidos (Kaplan e Norton, 1997). Propõem, então, um modelo de gestão contemplando também indicadores não-financeiros, possibilitando a avaliação dos ativos intangíveis tais como: relacionamento com clientes, processos de inovação, tecnologia da informação, capacidade e motivação dos colaboradores. De acordo com Kaplan e Norton (2001), os ativos intangíveis se tornaram, numa sociedade competitiva e mutável como a nossa, a principal fonte de vantagem competitiva, os principais responsáveis pelos resultados e sucesso a longo prazo.

Para Filguerias (2010), o termo balanced é utilizado para enfatizar o equilíbrio existente entre objetivos de curto e longo prazo, medidas financeiras e não financeiras, indicadores de ocorrência e tendência e entre perspectivas adotadas pelos sistemas de gestão, enquanto o termo scorecard é utilizado para ressaltar a forma pela qual os resultados dos períodos passaram a ser demonstrados.

De acordo com seus criadores, o $B S C$ é um sistema de gestão de desempenho que tem como foco a visão da empresa e as estratégias necessárias para atingi-la. Para tal, a avaliação do desempenho organizacional está desdobrada em quatro perspectivas: (i) perspectiva financeira: avaliação da estratégia de crescimento, rentabilidade e risco sobre a visão dos acionistas da organização. Isto pode ser aferido através de indicadores de retorno sobre investimento, riscos, ciclo de caixa, entre outros. (ii) perspectiva dos clientes (stakeholders): avaliação da criação de valor e diferenciação, sob a visão do cliente, utilizando como indicadores: satisfação, retenção, participação de mercado, entre outras. (iii) perspectiva dos processos internos: avaliação dos processos de negócio que criam satisfação para os clientes e acionistas, tais como logística interna e externa, qualidade, inovação, tempo de resposta, custos e lançamentos de novos produtos. (iv) perspectiva de aprendizado e crescimento: avaliação de aspectos relativos ao desenvolvimento de um clima propício à mudança organizacional, à inovação e ao crescimento.

No desenvolvimento de uma estrutura de causa para o efeito dos objetivos estratégicos, Kaplan e Norton (2004) criaram uma ferramenta denominada "Mapa Estratégico" visando traduzir as estratégias em termos operacionais. O mapa estratégico é uma representação visual da lógica da estratégia de uma organização, onde são conectados indicadores de desempenho aos objetivos estratégicos traçados. De acordo com Kaplan e Norton (1997), os indicadores de desempenho selecionados para um $B S C$ devem ser elementos de uma cadeia de relações de causa e efeito que comuniquem aos empregados da corporação o significado da estratégia estipulada e proporcionem aprendizado e crescimento.

\subsection{Pesquisas similares}

Na revisão da literatura internacional efetuada por meio do levantamento bibliométrico, foram selecionadas sete pesquisas similares como contribuição para este trabalho conforme tabela 1 . 


\begin{tabular}{c|c|c|c}
\hline$N^{o}$ & Ano & Autores & Título \\
\hline 1 & 2005 & Kettunen e Kantola & Implementation of strategies in continuing education \\
\hline 3 & 2006 & $\begin{array}{c}\text { Papenhausen e } \\
\text { Einstein }\end{array}$ & Implementing the Balanced Scorecard at a college of business. \\
\hline 4 & 2008 & Chen at all & $\begin{array}{r}\text { The application ofbalanced scorecard in the performance } \\
\text { evaluation of higher education }\end{array}$ \\
\hline 5 & 2011 & McClellan & $\begin{array}{r}\text { Beyond student learning outcomes: developing comprehensive, } \\
\text { strategic assessment plans for advising programmes }\end{array}$ \\
\hline 7 & 2012 & Schobel e Scholey & $\begin{array}{r}\text { Balanced Scorecards in education: focusing on financial } \\
\text { strategies }\end{array}$ \\
\hline
\end{tabular}

Tabela 1 - Relação de trabalhos similares levantados no estudo bibliométrico (Dados da pesquisa, 2013)

Pesquisando nestes trabalhos a utilização do $B S C$ em instituições de ensino superior, foram encontrados estudos sobre o quadro conceitual e viabilidade de aplicação da metodologia no ensino superior em Kettunen e Kantola (2004, 2005 e 2006); Kettunen (2005); McClellan (2006); Evans (2007); Asan e Tanyas (2007), Hess (2008) e Shobel e Scholey (2012).

Os pesquisadores Kettunen e Kantola (2005) apresentam a implantação uma política europeia de educação, a partir do denominado "Processo de Bologna". No planejamento do ensino superior do continente europeu é utilizado o $B S C$ para a comunicação e implementação das estratégias educacionais.

Aplicações práticas da metodologia em instituições de ensino superior são discutidas por Papenhausenattall (2006), na University of Massachusetts e McDevittattall (2008), na Fairfield University, ambas nos Estados Unidos. Já Strang (2010) apresenta a utilização do BSC na Austrália e Estados Unidos. Em Taiwan, Hsieh (2004) discute a utilização do BSC na gestão de bibliotecas universitárias e Chen et al (2006) apresenta a aplicação do BSC no Chin MinInstitute of Technology. Já Kettunen (2008) apresenta um quadro conceitual geral que pode ser usado para avaliar a qualidade e o desempenho institucional no ensino superior na Finlândia. Edvardsson (2008) estuda a aplicação do BSC na University of Akureyri na Islândia. Yuattall (2009) propõe a utilização do BSC para avaliação de desempenho de equipes acadêmicas na Malásia. Umashankar e Dutta (2007) discutem a validade da utilização do BSC em instituições de ensino superior da Índia e Nigam (2007) discute a utilização do BSC na gestão de cursos superiores à distância, na Turquia.

Dentre os relatos de aplicações do BSC no ensino superior, destacamos os trabalhos apresentados na tabela 2, com apontamento de indicadores de desempenho para a Perspectiva de Clientes. Neste estudo, denominou-se "Perspectiva dos Stakeholders", utilizado por Papenhausen e Einstein (2006) e Schobel e Scholey (2012), que melhor caracterizam os clientes internos e externos de uma instituição de ensino. 


\begin{tabular}{|c|c|c|c|c|c|c|c|}
\hline Indicadores de performance Perspectiva "Stakeholders"/ $N^{o}$ & 1 & 2 & 3 & 4 & 5 & 6 & 7 \\
\hline \% Satisfação dos estudantes & $\mathrm{x}$ & $\mathrm{x}$ & $\mathrm{x}$ & $\mathrm{x}$ & $\mathrm{x}$ & $\mathrm{x}$ & $\mathrm{X}$ \\
\hline \% Satisfação da família coma instituição & & $\mathrm{X}$ & & & & $\mathrm{X}$ & \\
\hline$\%$ de queixas dos estudantes / comunidade & & & $\mathrm{x}$ & & & & \\
\hline$\%$ Estudantes graduados empregados & $\mathrm{x}$ & $\mathrm{x}$ & $\mathrm{x}$ & $\mathrm{x}$ & & & \\
\hline \% Satisfação dos empresários comalunos graduados empregados & & & & $\mathrm{x}$ & & & \\
\hline$\%$ estudantes graduados empregados satisfeitos & $\mathrm{x}$ & & & & & & \\
\hline \% Estudantes graduados que permanecem da região & $\mathrm{x}$ & & & $\mathrm{x}$ & & & \\
\hline Serviços / produtos oferecidos à sociedade & & $\mathrm{x}$ & & $\mathrm{x}$ & & & \\
\hline Proporção de empresários entre estudantes graduados & & & & $\mathrm{x}$ & & & \\
\hline Imagem da instituição perante a sociedade & & $\mathrm{x}$ & $\mathrm{x}$ & & $\mathrm{x}$ & & \\
\hline Número de estudantes por programa & $\mathrm{x}$ & & & $\mathrm{x}$ & $\mathrm{x}$ & & \\
\hline Participação da instituição em projetos sociais & & & $\mathrm{x}$ & & & & \\
\hline \% estudantes em relação a população total de estudantes da região & & & & & & & $\mathrm{X}$ \\
\hline Nível de avaliação da instituição pelo órgão regulador & & & $\mathrm{x}$ & & & & \\
\hline Capacidade de atrair e formar estudantes de alta qualidade & & $\mathrm{X}$ & & & & & \\
\hline
\end{tabular}

Tabela 2 - Indicadores pela perspectiva de clientes nos estudos similares (Dados da pesquisa, 2013)

$\mathrm{Na}$ seleção de indicadores para a Perspectiva dos Clientes da experiência internacional observa-se unanimidade na seleção da "satisfação dos estudantes", bem como uma maior concentração de autores utilizando o nível de empregabilidade dos estudantes, quantidade de estudantes por programa e imagem da instituição perante a sociedade.

No capítulo seguinte, será apresentado o planejamento estratégico da instituição de ensino estudada e proposta a abordagem do $B S C$ para a definição de seus indicadores de desempenho.

\section{Metodologia}

O estudo foi iniciado pela pesquisa bibliométrica onde foram pesquisados artigos de 2002 até 2012 nas bases de dados ISI e SCOPUS no portal de Periódicos da CAPES. A partir de combinações das palavras chaves "balanced scorecard", "performance", "measur" e "high education", foram encontrados 478 artigos que, após exclusão das duplicações e exame do conteúdo, resultaram em 07 artigos com contribuições efetivas sobre o tema pesquisado. Metodologicamente, este trabalho classifica-se como uma pesquisa aplicada, com abordagem qualitativa. Trata-se de um estudo de caso, com pesquisa bibliográfica e documental. Utilizou-se a pesquisa bibliográfica a fim de possibilitar a pesquisa de fenômenos já estudados integrando-os ao estudo proposto (CERVO; BERVIAN, 2002).

O estudo de caso foi realizado numa Instituição de Ensino Superior Privada localizada na região de Florianópolis, Santa Catarina. Para Fonseca (2002) um estudo de caso pode ser caracterizado como um estudo de uma entidade bem definida como um programa, uma instituição, um sistema educativo, uma pessoa, ou uma unidade social. Visa conhecer em profundidade o como e o porquê de uma determinada situação que se supõe ser única em muitos aspectos, procurando descobrir o que há nela de mais essencial e característico. O levantamento dos dados e informações sobre a organização estudada assim como o conhecimento detalhado sobre o planejamento estratégico se deram por meio de pesquisas no endereço eletrônico da instituição e conversas com o diretor da faculdade.

Após análise das informações coletadas, foi proposto pelos autores um conjunto de indicadores voltados para a perspectiva de clientes, de acordo a metodologia proposta pelo $B S C$.

Por fim, os resultados encontrados neste estudo foram comparados com os resultados dos trabalhos levantados na pesquisa bibliométrica, servindo como base para elaboração da conclusão desta pesquisa. A seguir são apresentados os resultados e suas respectivas análises. 


\section{Apresentação e análise de resultado}

No presente capítulo serão apresentadas as características da instituição estudada e propostos o mapa estratégico e indicadores para a Perspectiva dos Stakeholders.

\subsection{A instituição privada de ensino superior analisada}

A Instituição de Ensino Superior Privada, base para este estudo, está localizada na cidade de Florianópolis em Santa Catarina. Atuante no mercado desde 1999, a Faculdade ALFA (assim denominada neste estudo para preservar o nome da Instituição) está inserida no mercado com cursos da área de Ciências Sociais Aplicadas, oferecendo cursos de Graduação, cursos Sequenciais e de Pós Graduação.

A instituição tem definida em sua estratégia a missão de despertar profissionais conscientes da importância na transformação da humanidade por meio da Formação e Qualificação Profissional de Alto Nível, a partir do desenvolvimento de capacidades e habilidades fundamentadas em abordagens e tecnologias de sustentabilidade, valores ético-sociais e sua interdependência com o ambiente, privilegiando a criação, inovação e aplicação do conhecimento. Como visão a Faculdade ALFA pretende se tornar uma Universidade integrada e de referência nas áreas de Gestão, Ciências Sociais Aplicadas, Ciências Jurídicas, Ciências Humanas e Ciências da Saúde, com forte presença regional, nacional e internacional; atuando com foco na educação, sustentabilidade e na responsabilidade social e ambiental.

A instituição apresenta basicamente três objetivos estratégicos: (i) Atingir rentabilidade considerada adequada pelos investidores para a prestação de serviço de educação de nível superior; (ii) fortalecer a imagem da instituição perante a comunidade onde está inserida; (iii) propiciar reconhecidamente um ensino de alta qualidade.

Em seu diagnóstico estratégico, a instituição tem a percepção do ambiente interno e externo conforme Quadro 1.

\begin{tabular}{|l|l|l|}
\hline \multicolumn{3}{|c|}{ Oportunidades Análise Externa } \\
\hline $\begin{array}{l}\text { Classes D e C em ascensão, crescimento da } \\
\text { economia Brasileira, crescimento da região de }\end{array}$ & $\begin{array}{l}\text { Expansão dos grandes grupos nacionais de educação, } \\
\text { crescimento da modalidade EAD (Ensino a distância), } \\
\text { Florianópolis, centralização das atividades } \\
\text { econômicas da cidade, valorização do curso superior } \\
\text { no país. }\end{array}$ & $\begin{array}{l}\text { lucacionais, carga tributária elevada para instituições } \\
\text { de educação privada. }\end{array}$ \\
\hline \multicolumn{2}{|c|}{ Pontos Fortes } \\
\hline $\begin{array}{l}\text { Bonálise Interna } \\
\text { de localização, agilidade em reagir às mudanças } \\
\text { atraentes, instalações de boa qualidade, boa imagem } \\
\text { junto à comunidade, quadro com 90\% dos } \\
\text { professores com 75\% pós-graduação stricto sensu. }\end{array}$ & $\begin{array}{l}\text { Limitação de espaço físico para crescimento, custo } \\
\text { elevado de locação das instalações, necessidade de mais } \\
\text { investimento em infraestrutura de informática, nível de } \\
\text { inadimplência dos alunos. }\end{array}$ \\
\hline
\end{tabular}

Quadro 1: Análise SWOT da instituição ALFA (Dados da pesquisa, 2013)

\subsection{Mapa estratégico proposto}

O BSC apresenta uma ligação de causa e efeito entre a missão da instituição de ensino e as estratégias de ação estabelecidas no processo de planejamento.

Esta ligação fica evidenciada no Mapa Estratégico onde são apresentados os ativos tangíveis e ativos intangíveis da instituição nas quatro perspectivas do $B S C$ (financeira, clientes, processos internos, crescimento e aprendizado), vinculados a indicadores mensuráveis que apontam a sua efetiva realização. O mapa estratégico apresentado na figura 1 ilustra como os ativos intangíveis são transformados em cliente tangíveis e estes, em resultados financeiros. 


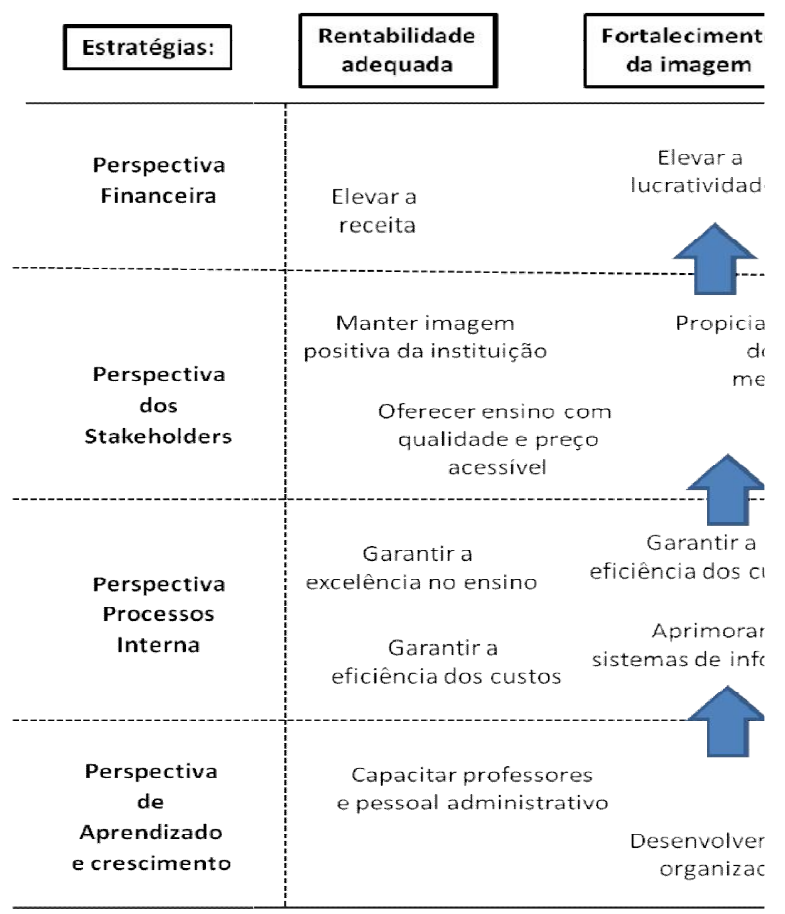

Figura 1- Mapa estratégico proposto para a Faculdade Alfa (elaborado pelos autores, 2013)

A partir do mapa estratégico definido, no item a seguir são discutidos indicadores para avaliação da Perspectiva dos Stakeholders (clientes) do Balanced Scorecard.

\subsection{Foco na perspectiva de clientes (stakeholders)}

O objetivo nesta perspectiva é aumentar a satisfação dos clientes internos e externos. Quando os clientes internos e externos estão satisfeitos com o desempenho acadêmico e serviços prestados, temos a promoção de uma boa imagem da instituição junto à sociedade e, consequentemente, $o$ crescimento da organização. Os objetivos elencados para esta Perspectiva e os indicadores definidos para seu acompanhamento podem ser visualizados na Tabela 3.

\begin{tabular}{l|l}
\hline \multicolumn{1}{c|}{ Objetivos } & \multicolumn{1}{c}{ Indicadores } \\
\hline Manter a Imagem Positiva da Instituição & $\begin{array}{l}\text { Índice de satisfação dos estudantes } \\
\text { Imagem da instituição perante a sociedade } \\
\text { Índice de satisfação dos empregados com a capacitação dos alunos graduados } \\
\text { Índice de transferências externas dos estudantes }\end{array}$ \\
\hline $\begin{array}{l}\text { Oferecer Ensino de Qualidade com preço } \\
\text { acessível }\end{array}$ & $\begin{array}{l}\text { Nível da avaliação da instituição perante órgão avaliador (MEC) } \\
\text { Comparação do valor da mensalidade com concorrentes diretos } \\
\text { Número de trabalhos científicos publicados }\end{array}$ \\
\hline $\begin{array}{l}\text { Propiciar inclusão e ascensão ao mercado de } \\
\text { trabalho }\end{array}$ & $\begin{array}{l}\text { Índice de empregabilidade dos estudante e alunos graduados } \\
\text { Índice de crescimento profissional entre os alunos graduados }\end{array}$ \\
\hline $\begin{array}{l}\text { Prestar serviços à comunidade } \\
\text { Quantidade de horas de atividades de ensino oferecidas à comunidade } \\
\text { Quantidade de horas de atividades de cidadania oferecidas à comunidade }\end{array}$ \\
\hline
\end{tabular}

Tabela 3 - Proposição de Indicadores pela "Perspectiva dos Stakeholders" para a Faculdade Alfa (elaborado pelos autores, 2013).

Os indicadores selecionados para esta perspectiva estão alinhados com os encontrados na experiência internacional relatada nos estudos levantados pela pesquisa bibliométrica deste trabalho. 
A "satisfação dos estudantes" está presente em todos os estudos de caso encontrados na revisão de literatura. Em uma instituição de ensino privada, onde os estudantes arcam com o custo dos serviços educacionais oferecidos, este indicador se torna de grande importância.

A “imagem da instituição perante a sociedade" está presente em Chen et al. (2006), McClellan (2011) e Papenhausen e Einstein (2006). Também pode ser percebida pelo "índice satisfação dos empregadores com a capacitação dos alunos graduados", proposto por Kettunen (2008).

Destaca-se também o "índice de empregabilidade dos estudantes", presente nos estudos de Kettunen e Kantola (2005), Kettunen (2008), Chen et al. (2006) e Papenhausen e Einstein (2006).

A "qualidade do ensino oferecida" pode ser percebida pelo resultado do "nível de avaliação da instituição pelo órgão avaliador", proposto por Chen et al. (2006) e pelo "número de trabalhos científicos" apresentados em congressos e / ou publicados em revistas especializadas.

A "demanda pela sociedade" dos serviços educacionais da instituição é aferida pela média de estudantes por programa oferecido pela instituição em Kettunen e Kantola (2005), Kettunen (2008), Chen et al. (2006) e Papenhausen e Einstein (2006). Para avaliar este aspecto na instituição analisada, foi sugerido o "índice de transferências externas dos estudantes".

A inserção da instituição em sua comunidade está representada pelos indicadores "quantidade de horas de ensino oferecidas à comunidade", lembrado por Kettunen ( 2008) e Papenhausen e Einstein (2006), bem como em "quantidade de horas de atividade de cidadania oferecidas a comunidade", citado por Chen et al. (2006).

Pela característica da instituição em trabalhar principalmente com um público de classe média, foram definidos também indicadores para assinalar se o nível de preços dos serviços educacionais não são superiores à média das demais instituições particulares de ensino superior da região. Este aspecto não foi abordado por nenhum dos autores estudados.

\subsection{Ponto de situação: ações a implementar na faculdade alfa}

A Faculdade Alfa tem um bom sistema de comunicação educacional e administrativo, com informações em arquivo que permitem avaliar o histórico de desempenho da instituição. No entanto, como não tem ainda definido um processo formal de acompanhamento estratégico, existe a necessidade de estudos adicionais para definição do histórico dos indicadores selecionados e definição de patamares a serem atingidos nos próximos anos.

A seguir, são demonstrados o ponto de situação e as ações a desenvolver para cada um dos itens dos objetivos da perspectiva dos stakeholders.

\section{(i) Manter a imagem positiva da instituição:}

Satisfação dos estudantes: a faculdade conta com o trabalho institucionalizado da Comissão Permanente de Avaliação - CPA, que promove avaliação anual da estrutura, direção, ensino e desempenho dos professores. Seus resultados são amplamente divulgados na intranet e afixados em local visível na instituição. Ações a implementar: levantar o histórico das avaliações e definir metas para os próximos anos.

Imagem da Instituição perante a sociedade e índice de satisfação dos empregadores com a capacitação dos alunos graduados: neste quesito a faculdade apresenta grande carência de informações estratégicas. A instituição não possui nenhum estudo de empregabilidade de seus estudantes, nem dos segmentos profissionais onde seus formandos atuam. Não existe um canal de comunicação formal de acompanhamento da vida profissional dos antigos alunos. Com isto, a faculdade tem dificuldade em diagnosticar ajustes necessários em seus programas de curso, bem como de perceber necessidades pontuais que poderiam ser satisfeitas com a oferta de cursos de extensão à comunidade. Ações a implementar: instituir uma pesquisa anual de acompanhamento dos alunos formandos nos primeiros cinco anos de atividade. Implantar uma pesquisa de opinião 
junto aos empregadores de alunos da instituição. Para esta iniciativa há a necessidade da anuência dos alunos envolvidos.

Índice de transferência externa de estudantes: refere-se ao histórico de alunos que ingressaram na faculdade, transferidos de outras instituições, bem como das solicitações de transferências externas. Ações a implementar: os dados históricos estão disponíveis nos sistemas de informação. Elaborar acompanhamento por curso oferecido.

(ii) Oferecer ensino de qualidade com preço acessível:

Avaliação da instituição pelo MEC: este é o indicador mais importante no que se refere ao reconhecimento da qualidade da instituição. Sua aplicação é disciplinada por regulamentação do Ministério da Educação e Cultura.

Comparação do valor da mensalidade com concorrentes diretos: este acompanhamento, atualmente, é efetuado informalmente. Ações a implementar: sistematizar, de forma a obter-se a evolução histórica. Definir nível relativo de preços em relação aos concorrentes.

Número de trabalhos científicos publicados: refere-se a artigos de autoria de estudantes e professores em revistas científicas. Ações a implementar: incentivar e orientar os alunos a elaborarem e publicarem artigos científicos nos trabalhos de conclusão do curso.

(iii) Propiciar a inclusão e ascensão dos estudantes no mercado de trabalho:

Neste tópico, temos os indicadores "Índice de empregabilidade dos estudantes e alunos graduados" e "Índice de crescimento profissional entre os alunos graduados". Estas informações são de grande importância estratégica para a compreensão das necessidades do corpo discente bem como para avaliar o ganho de qualidade de vida dos alunos que passaram pela instituição. Ações a implementar: instituir esta pesquisa entre alunos e formandos dos últimos cinco anos.

(iv) Prestação de serviços à comunidade:

Quantidade de horas de ensino oferecidas à comunidade: Este indicador exige o desenvolvimento da sensibilidade dos diretores da instituição para com as necessidades da comunidade. Um crescimento do número de horas de cursos abertos ao público em geral demonstra a proatividade da instituição em relação ao ambiente onde atua. Ações a implementar: definir metas após análise das pesquisas junto à sociedade, previstas nos itens (i) e (iii).

Quantidade de horas de atividades de cidadania oferecidas à comunidade: Atualmente a instituição realiza diversas ações pontuais de filantropia, por iniciativa de seus alunos e professores. Ações a implementar: implantar programas coordenados de ação social. Identificar junto aos órgãos de ação social que atividades poderiam ser desenvolvidas por alunos da instituição como "atividade complementar".

O estudo em questão abordou um tema pouco encontrado na pesquisa bibliométrica previamente realizada sobre Avaliação de Desempenho em Instituições de Ensino Superior. Como semelhança é possível citar o estudo do BSC como ferramenta de avaliação de desempenho e a elaboração do mapa estratégico para instituições de ensino superior. Entretanto os artigos encontrados não abordaram a perspectiva de clientes voltados para Instituições privadas, principal diferencial deste estudo.

\section{Considerações finais}

A expansão recente do ensino superior no Brasil foi atrelada basicamente às instituições privadas. As instituições públicas, com uma estrutura organizacional muito pesada e processo decisório lento (principalmente por serem entidades públicas) não conseguem atender a demanda crescente por vagas no ensino superior. Desta forma este trabalho buscou estudar uma instituição de ensino superior privada localizada na cidade de Florianópolis, SC. Este estudo se propôs a lançar um olhar em particular, sobre o que seria a excelência em serviços educacionais percebido pela sociedade. 
Se por um lado uma instituição privada tem, normalmente, limitações de recursos e de infraestrutura, sua capacidade de adequação às necessidades da sociedade pode ser bastante ágil em decorrência do processo de gestão privada.

A adoção de um instrumento de planejamento como o Balance Scorecard traz uma visão diferenciada de como avaliar a postura estratégica da instituição. A definição de indicadores de performance para a Perspectiva dos Stakeholders deixaram claro a necessidade de aproveitar oportunidades estratégicas até então ignoradas pela instituição estudada. Indicadores do nível de empregabilidade, ascensão profissional e satisfação dos empregadores, por exemplo, fornecerão importantes informações sobre as necessidades da sociedade e manterão um forte vínculo entre os formandos e a sua instituição de origem.

Assim sendo, o problema de pesquisa formulado e objetivo geral deste estudo foram alcançados com a proposição e reflexão dos indicadores adequados para uma IES privada com as características da instituição estudada.

Como limitações deste estudo, aponta-se que os indicadores levantados para a Faculdade Alfa não devem ser replicados automaticamente para outras instituições em função das particularidades de cada organização. Vale destacar que a implantação de um sistema de acompanhamento dos estudantes envolve custo e tempo para execução, sendo a direção da organização responsável por avaliar o custo benefício do projeto.

Como incentivo para trabalhos futuros sugere-se a implantação desta proposta de avaliação de desempenho na IES, além do desenvolvimento das demais perspectivas propostas pelo Balanced Scorecard.

\section{Referências}

BARREYRO, Gladys Beatriz. Mapa do ensino superior privado. MEC, Ministério da Educação, INEP, Instituto Nacional de Estudos e Pesquisas Educacionais Anísio Teixeira, 2008.

BRASIL, M.E.C. Ministério da Educação. Censo da Educação Superior 2011. Qualidade da Educação Superior. Disponível em: http://portal.mec.gov.br/index.php?option=com_docman\&task. Acesso em: 18 jan. 2013.

CHEN, Shun-Hsing; YANG, Ching-Chow; SHIAU, Jiun-Yan. The application of balanced scorecard in the performance evaluation of higher education. The TQM Magazine, $\mathrm{v} .18, \mathrm{n}$. 2, p. 190-205, 2006.

COSTA, Danilo de Melo; BARBOSA, Francisco Vidal; GOTO, Melissa Midori Martinho. O novo fenômeno da expansão da educação superior no Brasil. REUNA, v. 16, n. 1, 2011.

EDVARDSSON, Ingi Runar; OSKARSSON, Gudmundur Kristjan. Distance education and academic achievement in business administration: The case of the University of Akureyri. The International Review of Research in Open and Distance Learning, v. 9, n. 3, 2008.

EVANS, James R. Impacts of information management on business performance. Benchmarking: An International Journal, v. 14, n. 4, p. 517-533, 2007.

DE ALMEIDA FILGUEIRAS, Aline; DE SOUZA BARROS, Luana Paula; GOMES, Josir Simeone. O processo de implantação do balanced scorecard em uma empresa estatal brasileira: o caso Petrobras. REGE Revista de Gestão, v. 17, n. 1, p. 45-57, 2010. 
FRANCESCHINI, Fiorenzo; TURINA, Elisa. Quality improvement and redesign of performance measurement systems: an application to the academic field. Quality \& Quantity, v. 47, n. 1, p. 465-483, 2013.

GALVÃO, Henrique Martins; CORRÊA, Hamilton Luiz; ALVES, José Luiz. Modelo de avaliação de desempenho global para instituição de ensino superior. Revista de Administração da UFSM, v. 4, n. 3, p. 425-441, 2011.

HESS, Frederick M.; FULLERTON, Jon. The numbers we need: Bringing balanced scorecards to education data. Phi Delta Kappan, v. 90, n. 9, p. 665-669, 2009.

HSIEH, Ling-Feng; CHIN, Jiung-Bin; WU, Mu-Chen. The performance indicators of university e-library in Taiwan. Electronic Library, The, v. 22, n. 4, p. 325-330, 2004.

KAPLAN, Robert S.; NORTON, David P. Balanced Scorecard: a estratégia em ação. Rio de Janeiro: Campus, 1997.

KAPLAN, Robert S.; NORTON, David P. Organização orientada para a estratégia. Rio de Janeiro: Campus, 2001.

KETTUNEN, Juha. A conceptual framework to help evaluate the quality of institutional performance. Quality Assurance in Education, v. 16, n. 4, p. 322-332, 2008.

KETTUNEN, Juha. Implementation of strategies in continuing education. International Journal of Educational Management, v. 19, n. 3, p. 207-217, 2005.

KETTUNEN, Juha. Strategic planning of regional development in higher education. Baltic Journal of Management, v. 1, n. 3, p. 259-269, 2006.

KETTUNEN*, Juha. The strategic evaluation of regional development in higher education. Assessment \& Evaluation in Higher Education, v. 29, n. 3, p. 357-368, 2004.

KETTUNEN*, Juha. The strategic evaluation of regional development in higher education. Assessment \& Evaluation in Higher Education, v. 29, n. 3, p. 357-368, 2004.

KETtUNEn, Juha; KANTOLA, Mauri. The implementation of the Bologna Process. Tertiary Education and Management, v. 12, n. 3, p. 257-267, 2006.

MCCLELLAN, Jeffrey L. Beyond student learning outcomes: developing comprehensive, strategic assessment plans for advising programmes. Journal of Higher Education Policy and Management, v. 33, n. 6, p. 641-652, 2011.

MCDEVITT, Roselie; GIAPPONI, Catherine; SOLOMON, Norman. Strategy revitalization in academe: a balanced scorecard approach. International Journal of Educational Management, v. 22, n. 1, p. 32-47, 2008.

MINISTÉRIO DA EDUCAÇÃO. Censo da Educação Superior 2011. Disponível em: http://portal.mec.gov.br/index.php?option=com_content\&view $=$ article\&id=18153. Acesso em: 21 jan. 2013.

NIGAM, Amrita; JOSHI, Vibha. Science education through open and distance learning at higher education level. Turkish Online Journal of Distance Education, v. 8, n. 4, p. 20-33, 2007. 
OTTO, Ann K.; NOVIELLI, Karen; MORAHAN, Page S. Implementing the logic model for measuring the value of faculty affairs activities. Academic Medicine, v. 81, n. 3, p. 280-285, 2006.

PAPENHAUSEN, Chris; EINSTEIN, Walter. Implementing the Balanced Scorecard at a college of business. Measuring Business Excellence, v. 10, n. 3, p. 15-22, 2006.

RAMOS, Alexandre M.; LOBO, Eduardo. Estratégias Genéricas de Competição Praticadas por IES Privadas de Pequeno Porte. X Coloquio Internacional sobre Gestion Universitaria em America del Sur, 2010.

RIGBY, Darrell; BRIDELLI, Stefano; ALVES, Vera. Ferramentas de gestão 2003. São Paulo: Bain \& Company, 2003.

SCHOBEL, Kurt; SCHOLEY, Cam. Balanced Scorecards in education: focusing on financial strategies. Measuring Business Excellence, v. 16, n. 3, p. 17-28, 2012.

SOARES, Maria Susana Arrosa. Educação superior no Brasil. Capes, 2002.

STRANG, Kenneth David. Education balanced scorecard for online courses: Australia and us best-practices. Journal of Cases on Information Technology (JCIT), v. 12, n. 3, p. 45-61, 2010.

YU, May Leen et al. The e-balanced scorecard (e-BSC) for measuring academic staff performance excellence. Higher Education, v. 57, n. 6, p. 813-828, 2009. 